\title{
CORRELATOS E VARIAÇÕES NA PERCEPÇÃO DO SUPORTE ORGANIZACIONAL E GESTÃO DO CONHECIMENTO EM TRABALHẢDORES DE ORGANIZAÇÕES PÚBLICAS E PRIVADAS EM NATAL-RN
}

\section{CORRELATES AND VARIATIONS IN THE PERCEPTION OF ORGANIZATIONAL SUPPORT AND KNOWLEDGE MANAGEMENT IN WORKERS OF PUBLIC AND PRIVATE ORGANIZATIONS IN NATAL-RN}

\author{
Nilton Soares Formiga ${ }^{1}$, Juliana Bianca Maia Franco², Sarah Ruth Araújo Grangeiro³, Heitor César Costa
} Oliveira ${ }^{4}$, Ionara Dantas Estevam ${ }^{5}$

\author{
Submetido em: 19/04/2021 \\ Aprovado em: 10/05/2021
}

e24213

\section{RESUMO}

Os estudos na área organizacional e do trabalho são abordados a luz do discurso da globalização e o processo no mundo do trabalho e o quanto as mudanças ocorridas neste contexto tem influenciado no comportamento do trabalhador. Estas condições não estão limitadas ao contexto geográfico e político, mas, tem revelado interesse por parte dos pesquisadores em estudos sobre os efeitos que essas mudanças podem gerar no binômio trabalhador-organização e desenvolvimento de estratégias para se manterem competitivas. Este artigo tem como objetivo verificar a influência do suporte organizacional sobre a gestão do conhecimento. Participaram do estudo, 208 trabalhadores, homens e mulheres, acima de 18 anos, de organizações públicas e privadas na cidade de Natal-RN, os quais, responderam um questionário sociodemográfico, a escala de suporte organizacional e gestão do conhecimento. Além da identificação de que as escalas são fidedignas, observou-se que o suporte organizacional se relacionou positivamente, seja em sua pontuação total dos escores entre os construtos, seja na especificidade dos fatores da gestão do conhecimento; numa análise de variância, destaca-se que os sujeitos que tiveram escores mais altos no suporte organizacional, pontuaram, também, mais alto na gestão do conhecimento. Com isso, os resultados desta dissertação não apenas foram confirmados, mas, também, têm sua importância não apenas para refletir práticas para o ser humano $(\mathrm{RH})$, mas também de política organizacional de produtividade e qualidade de vida do trabalhador em seu ambiente laboral.

PALAVRAS-CHAVE: Gestão do Conhecimento. Suporte Organizacional. Organizações Públicas e Privadas.

\section{ABSTRACT}

Studies in the organizational and work areas are approached in the light of the globalization discourse and the process in the world of work and how much the changes that have occurred in this context have influenced the behavior of the worker. These conditions are not limited to the

\footnotetext{
1 Mestrado em Psicologia (Psicologia Social) pela UNIVERSIDADE FEDERAL DA PARAÍBA. Em 2010 doutorou-se, também, na UNIVERSIDADE FEDERAL DA PARAÍBA, na pós-graduação em Psicologia Social. Atualmente, é professor/pesquisador no programa de pós-graduação (mestrado) em psicologia organizacional e do trabalho na Universidade Potiguar (UNP) /Laureate International Universities.

2 Universidade Potiguar/Laureate International Universities

3 Universidade Potiguar/Laureate International Universities

4 Universidade Potiguar/Laureate International Universities

5 Psicóloga, possui Doutorado em Psicologia Social pela Universidade Federal da Paraíba, Mestrado em Psicologia pela Universidade Federal da Paraíba, Especialização em Desenvolvimento Infantil e seus Desvios pela Universidade Federal da Paraíba, Especialista em Psicologia Clínica e Psicologia do Trânsito pelo Conselho Federal de Psicologia, Graduação em Psicologia pelo Centro Universitário de João PessoaUNIPÊ, Graduação em Licenciatura em Psicologia pelo Centro Universitário de João Pessoa-UNIPÊ. Professora e Pesquisadora da Pós-Graduação Stricto Sensu, Lato Sensu e Graduação de Psicologia da Universidade Potiguar - RN. Professora da Faculdade Mauricio de Nassau - RN.
} 


\section{RECIMA21 - REVISTA CIENTÍFICA MULTIDISCIPLINAR ISSN 2675-6218}

Nilton Soares Formiga, Juliana Bianca Maia Franco, Sarah Ruth Araújo Grangeiro, Heitor César Costa Oliveira, lonara Dantas Estevam

geographical and political context but have shown interest on the part of researchers in studies on the effects that these changes can have on the binomial worker-organization and the development of strategies to remain competitive. This article aims to verify the influence of organizational support on knowledge management. 208 workers, men and women, over 18 years old, from public and private organizations in the city of Natal-RN participated in the study, who answered a sociodemographic questionnaire, the scale of organizational support and knowledge management. In addition to the identification that the scales are reliable, it was observed that organizational support was positively related, either in its total score of scores between the constructs, or in the specificity of knowledge management factors; in an analysis of variance, it is highlighted that the subjects who had higher scores in organizational support, also scored higher in knowledge management. With that, the results of this dissertation were not only confirmed, but also have their importance not only to reflect practices for the human being $(H R)$, but also of the organizational policy of productivity and quality of life of the worker in his work environment.

KEYWORDS: Knowledge Management. Organizational Support. Public and Private Organizations.

\section{INTRODUÇÃo}

Os estudos na área organizacional e do trabalho, seja no campo das ciências humanas ou sociais, têm sido refletidos à luz do discurso da globalização e o processo no mundo do trabalho, como as mudanças ocorridas neste contexto têm influenciado no comportamento do trabalhador, na maioria das vezes, associado a exigente atitude de competitividade, inovação e a aderência às novas concepções sobre empregabilidade; tais condições não estão limitadas ao contexto geográfico e político, mas, também, vem se revelando interesse dos pesquisadores, em estudos sobre os efeitos que essas mudanças, justamente, por serem imprevisíveis, 'impõem' ao binômio trabalhador-organização e ao desenvolvimento de estratégias para se manterem competitivas (Formiga, Fernandes, Freire, Franco, Oliveira, Prochazka, Valin, Grangeiro, \& Estevam, 2021).

Essas situações interferem de forma direta na estrutura e funcionalidade da organização, especialmente, em relação a execução das atividades e tarefas realizadas pelo recurso humano que compõem as equipes multifuncionais de trabalho (Zanelli, Borges-Andrade, \& Bastos, 2004; Costa, 2019). É com base nestas concepções que a tríade organizações-trabalho-gestão tem recebido e vem desenvolvendo uma maior atenção não apenas na análise, compreensão e manutenção de variáveis, as quais, atribuem uma subjetividade avaliativa (por exemplo, avaliação do binômio saúde-adoecimento no trabalho, mas, também, avaliações de medidas dos eventos emocionais nos trabalhadores que interferem na criatividade e inovação organizacional, expectativa no apoio organizacional e o bem-estar mental do trabalhador, etc.), inibindo 0 desenvolvimento e a produtividade na afinidade organização-trabalho-indivíduo (Borges-Andrade, Abbad, \& Mourão, 2006; Leite \& Nogueira, 2017).

Sendo assim, os estudos contemporâneos sobre as organizações e trabalho têm se concentrado na avaliação da conduta e comportamento dos funcionários no seu ambiente laboral, pois, são elementos de extrema importância para o desempenho e competitividade 


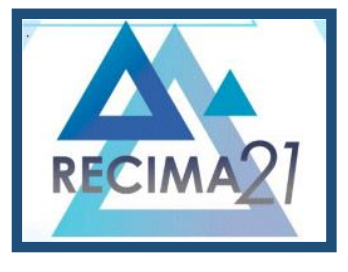

\section{RECIMA21 - REVISTA CIENTÍFICA MULTIDISCIPLINAR ISSN 2675-6218}

organizacional, consecutivamente, um fator crucial para a retenção de lucros das empresas (cf. Siqueira, 2005; Siqueira, 2014).

Tais concepções justificam a preocupação quanto à forma de gerenciar o capital humano buscando promover o comprometimento e envolvimento de seus funcionários; principalmente, às condutas contraproducentes, antiéticos, entre outros, os quais, poderão estar pautados, dentre as inúmeras variáveis no campo da psicologia organizacional e do trabalho, na forma de apoio que a organização ofereça ao funcionário, bem como, a maneira com ele organizada e gera conhecimentos destinado a uma melhor solução de problema e tomada de decisão capaz de contribuir para uma melhor produtividade e desenvolvimento organizacional.

Neste contexto, para Siqueira \& Padovan (2008), a palavra suporte associa-se a algo ou alguém que nos ofereça apoio, sustentação e proteção, sendo o suporte social um atenuador de problemas. Neste mesmo sentido, numa perspectiva de comportamento organizacional, (Eisenberger, Huntington, Hutchinson, \& Sowa, 1986). Define suporte organizacional quanto as argúcias do trabalhador sobre a qualidade do tratamento que recebe da organização em resposta ao seu esforço no desenvolvimento de suas funções laborais.

As percepções sobre a teoria do suporte organizacional convergem em vários aspectos na sua conceituação. Em coerência com a abordagem teórica de Eisenberger, Huntington, Hutchinson, \& Sowa, (1986), autores como Pereira, Formiga \& Estevam (2019) afirmam que quanto mais flexível for a organização no que se refere ao desempenho das tarefas, e de comum acordo com os trabalhadores, possivelmente terá melhor produtividade e como resultado, alcançará menor possibilidade de sofrimento do trabalhador (Formiga, Fernandes, Freire, Franco, Oliveira, Prochazka, Valin, Grangeiro, \& Estevam, 2021).

De igual forma, o estudo apresentado por Bertocello \& Borges-Andrade (2015) evidencia que a percepção do apoio organizacional apresenta interferência na saúde mental do trabalhador em áreas como depressão, síndrome de Burnout, estresse e ansiedade, afirmando, portanto, que uma boa percepção de suporte organizacional, provavelmente trará maiores fatores de proteção para a saúde do trabalhador.

Neste aspecto, a busca da estruturação mental no trabalho traz consequências positivas para a instituição, haja vista que a presença do sofrimento sugere que alguma coisa não está reagindo bem e impulsiona o indivíduo a buscar mudanças, sejam elas de caráter social ou pessoal (Torres, Chags, Moreira, Barreto, \& Rodrigues, 2013).

Desta forma, o suporte organizacional pode ser compreendido como uma maneira de contrato psicológico firmado entre as partes, principalmente por suas funções ficarem em torno das expectativas de troca e benefícios mútuos (Formiga, Fleury, \& Souza, 2014; Formiga, Freire, Azevedo, Nascimento, Franco, Oliveira, Prochazka, \& Grangeiro, 2021).

A manutenção do bem-estar mental do trabalhador é de extrema importância para a empresa, pois a produtividade e os lucros podem apresentar associação direta com o alívio do 


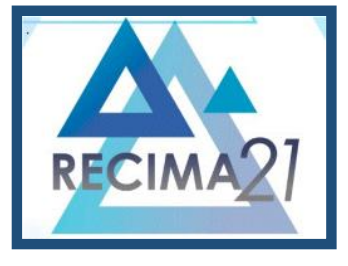

\section{RECIMA21 - REVISTA CIENTÍFICA MULTIDISCIPLINAR ISSN 2675-6218}

indivíduo. Isto significa que a manutenção do contrato psicológico entre trabalhador e organização, com ações que potencializam a saúde mental e aumentem a percepção do apoio organizacional do trabalhador, pode ser uma maneira ímpar de promover a efetividade e a eficácia da organização (Bertocello \& Borges-Andrade, 2015).

Formiga, Freire, Batista \& Estevam (2017), em seu estudo com 282 trabalhadores de empresas privadas e públicas do município de João Pessoa/PB, no Brasil, cujo objetivo foi verificar a relação entre suporte organizacional e autoestima, concluíram que o suporte organizacional se correlacionou de forma positiva com a autoestima positiva, tanto na amostra total quanto na amostra das organizações públicas, mas o mesmo não aconteceu com a amostra das organizações privadas. Outro resultado que encontraram foi a relação negativa do suporte organizacional com a autoestima negativa em todas as amostras (amostra geral, amostra da empresa pública e da privada).

O conceito de suporte organizacional possibilita análises mais profícuas de sua relação com outros conceitos e variáveis do comportamento organizacional, por exemplo, o conceito de capital psicológico, haja vista que este remete-se a um estado de desenvolvimento psicológico relacionado com o resultado e o desempenho no trabalho, como também com a satisfação e o comprometimento (Formiga, Freire, Azevedo \& Faria, 2020).

Já faz um tempo que o tema sobre Gestão do Conhecimento tem sido abordado; muitas áreas cientificas (Administração, Psicologia e Sociologia da organização etc.) e o meio organizacional vem se preocupando com esse espaço de avaliação, pois, alguns estudos associam a gestão do conhecimento aos processos organizacionais sobre o desempenho organizacional, inovação, criatividade (Fandiño, Formiga \& Menezes, 2018).

Desta maneira, a fim de o processo de compartilhamento de ativos tangíveis e intangíveis seja efetivo, segundo McInerney \& Ali (2006), há a obrigação de que existam clima e ambiente interno que facilitem interações humanas a partir da confiança mútua, da colaboração e cooperação, do mérito e de cultura organizacional centrada em regras claras que são reconhecidas, seguidas e valorizadas de forma objetiva e subjetiva para o funcionário e equipes.

Para tanto, é preciso, na concepção de Mohapatra, Agrawal \& Satpathy (2016), focar em três níveis de gestão do conhecimento (o individual, o de grupo - comunidade de práticas - e o organizacional); teoricamente, na expectativa de que a organização funcione de forma produtiva. Afinal, cada nível deste, demanda uma abordagem quanto ao conhecimento e sua aplicação na dinâmica organizacional.

Assim sendo, segundo Miranda (2006), salientar tais níveis, faz com que reflita a implementação de um sistema de gestão do conhecimento estratégico, o qual tem sua base no modelo sistêmico, que contempla desde a cultura organizacional, ao registro de conhecimento explícito em banco de dados específicos a aqueles chamados de filtradores e provedores de 


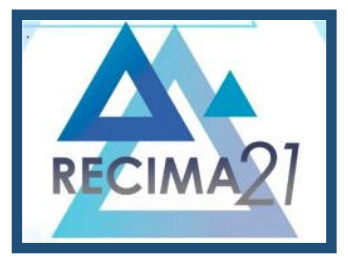

\section{RECIMA21 - REVISTA CIENTÍFICA MULTIDISCIPLINAR ISSN 2675-6218}

informação, bem como, na identificação de pessoas-chave dentro e fora da organização quanto função de qualidade produtiva.

Azevedo (2018) enfatiza que o ambiente que fomenta um espaço organizacional está em constante mudança, alterando-se a cada geração, e esse é o motivo que faz surgir novas dinâmicas dentro de cada cenário organizacional. Essa perspectiva de constante mudança imprime uma necessidade social e organizacional que obriga as empresas públicas e privadas a desenvolverem novas fontes de recursos. Com tudo, a sociedade se torna mais exigente e o mercado se torna mais competitivo. Este é um processo que ocorre, dentre outros fatores, graças ao acesso massificado às possíveis combinações entre os recursos, o que gera novos processos, novas tecnologias e novas práticas de gestão.

Sobre Gestão de Conhecimento, destaca-se que a gestão das organizações, até a década de 1980, tinha entre os seus principais fatores o acesso à mão de obra barata, aos recursos naturais e ao capital financeiro. Pouca importância era atribuída ao conhecimento enquanto parte do ativo, uma forma de capital ou até um bem organizacional. Entretanto, no final desta mesma década e durante a década posterior, as características de gestão se desenvolvem para outras temáticas que ainda se destacam hoje, sobretudo, a gestão do conhecimento (Formiga, Silva, Silva, Firmino, Santos, Azevedo \& Martins, 2020).

Isto posto, compreende-se que a Gestão do Conhecimento pode ser compreendida como uma disciplina que visa projeção e programação de um sistema que visa identificar, captar e fazer o compartilhamento do conhecimento comprimido de um ambiente organizacional, que esse conhecimento tenha o poder de transformação de valor para a empresa. Por apresentar a capacidade de conversão em valor para uma organização, o conhecimento tem um significado amplo, pois pode contribuir de maneira efetiva para o alcance dos objetivos perseguidos pela organização (Almeida, Formiga, Junior \& Sousa, 2020). Sendo assim, a partir dessas reflexões, o presente estudo tem como objetivo averiguar a extensão da percepção do suporte organizacional na gestão do conhecimento em labutadores de organização pública e privada no Brasil.

\section{MÉTODO \\ AMOSTRA}

Tratar-se de uma pesquisa quantitativa, do tipo representado, exploratório e correlacional, para o qual, participaram pessoas da população geral na Cidade de Natal-RN. A amostragem referente foi avaliada no pacote estatístico G Power 3.1 (Faul, Erdfelder, Lang, \& Buchner, 2007).

A coleta de dados foi realizada de forma não probabilística, através do método bola de neve, pois, considerou-se a disponibilidade e interesse da respondente em participar da pesquisa.

Para atender ao objetivo principal deste estudo, a partir dos critérios estabelecidos no Gpower, uma amostra de 208 trabalhadores revelou ser suficiente para o estudo, a qual, 


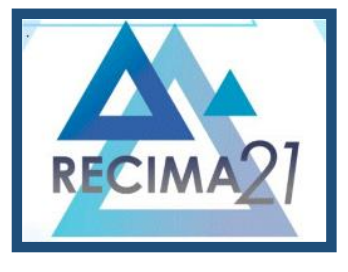

\section{RECIMA21 - REVISTA CIENTÍFICA MULTIDISCIPLINAR ISSN 2675-6218}

Nilton Soares Formiga, Juliana Bianca Maia Franco, Sarah Ruth Araújo Grangeiro, Heitor César Costa Oliveira, lonara Dantas Estevam

apresentou os seguintes indicadores estatísticos em relação a qualidade da amostra: $t \geq 1,98, \pi \geq$ $0,93, p<0,05$.

Essa amostra não somente é suficiente para a execução do estudo em questão, mas, também, revela condição para a concretização dos cálculos estatísticos relacionados ao objetivo da pesquisa. Quanto aos critérios de inclusão para os partícipes da observação, são destacados os seguintes: idade acima de 18 anos, pessoas dispostas a colaborar com a pesquisa respondendo ao questionário enviado por meio de plataforma digital, pertencer a qualquer classe social e receber mensalmente acima de um salário-mínimo.

\section{INSTRUMENTOS DE PESQUISA}

Os participantes responderam aos seguintes questionários (ver anexo):

Escala de Percepção de Suporte Organizacional (EPSO). Trata-se de uma escala composta por 9 itens, desenvolvida por Eisenberger et al (1986) e adaptada e validada para 0 contexto brasileiro por Siqueira (1995). Ela tem como objetivo aferir o quanto os indivíduos de organizações públicas e/ou privadas percebem o quanto estas se preocupam com o bem-estar do trabalhador. Para isso, o presente construto é mensurado através de uma escala de resposta de sete pontos, do tipo Likert, na qual, o respondente deverá indicar o quanto cada um dos itens corresponde a sua realidade na organização, devendo indicar na seguinte variação de resposta 1 = discordo totalmente, a 7 = concordo totalmente sobre o fenômeno abordado.

A título de atualização, no Brasil, os estudos desenvolvidos por Fleury, Formiga, Souza \& Souza (2017) e Formiga, Miranda, Araújo, Pastana \& Mafra (2018), com trabalhadores em diferentes organizações na região nordeste e sudeste com esta escala, não apenas revelou alfa próximo ao notado por Siqueira (1995), mas, através da análise fatorial confirmatória, a estrutura fatorial original foi, mais uma vez, comprovada revelando indicadores psicométricos confiáveis.

Escala de Gestão do Conhecimento (EGC) - Essa medida, conforme Pais (2014), apresenta um conjunto de itens que abrange a totalidade de práticas organizacionais vinculadas a processos de gestão do conhecimento. A versão reduzida desta escala é composta por 22 Itens distribuídos em quatro fatores:

- O Fator 1, orientação cultural para o conhecimento (7 itens), indica a orientação para práticas, regras, normas e a procedimentos instituídos que precisam ser seguidos. Indica referencial comum e memória coletiva. Ressalta conjunto partilhado de valores que é reconhecido.

- O Fator 2, orientação competitiva (4 itens), se refere à orientação para o exterior por parte da organização. É a lógica do conhecimento inserida na competitividade, no desempenho comparativo e no contexto de clientes e concorrentes. Permite a gestão estratégica do conhecimento, a adaptação ao ambiente externo e a vantagem competitiva.

- O Fator 3, práticas formais de gestão do conhecimento (6 itens), integra os esforços organizacionais concebidos em torno de processos formalmente instituídos na dimensão do 


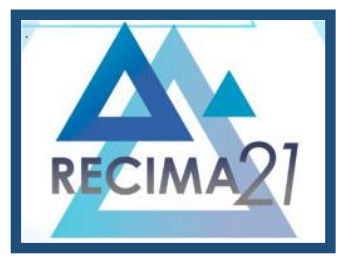

\section{RECIMA21 - REVISTA CIENTÍFICA MULTIDISCIPLINAR ISSN 2675-6218}

conhecimento, em especial, explícito. É o contíguo de práticas que visa criar, bem como permitir a aquisição de conhecimento. Objetiva-se preservar, partilhar e utilizar o conhecimento em produtos e serviços. Permite a mudança e o desenvolvimento organizacional. Viabiliza a tomada de iniciativa e a inovação.

- O Fator 4, práticas informais de gestão do conhecimento (5 itens), traduz as interações que ocorrem na organização e que facilitam a construção social do conhecimento. Emergem nas práticas discursivas e na criação de linguagem comum e coletiva. Há a ênfase no conhecimento de natureza tácita a datar do contato direto e presencial entre indivíduos. Trata-se de gestão simbólica do conhecimento que permite a atribuição de sentido.

A consistência da EGC numa amostra de trabalhadores brasileiros. Considerando que à proporção que em questão é organizada em quatro fatores, conseguiu-se uma análise fatorial confirmatória, a partir da qual, observou-se apontadores psicométricos que corresponderam ao que é determinado pela literatura, revelando com isso, que consistência e atributo psicométricos da escala, com todos os fatores se relacionando positivamente entre eles (Grangeiro \& Formiga, 2021).

No estudo desenvolvido por Almeida, Formiga, Junior \& Sousa (2020) com enfermeiros em unidades de referências na Paraíba, que realizam quimioterapia, avaliando a gestão do conhecimento, a presente escala revelou indicadores psicométricos confiáveis, atendendo com isso, aos critérios de qualidade sobre escalas avaliadoras de comportamento humano em outros profissionais e de que a existência desses quatro fatores é fidedigna.

Além desse instrumento, um questionário com a caracterização sociodemográfica foi incluído (aspectos relacionados a sexo, idade, renda, especialização profissional, tempo de servidão e tempo de formado, etc.).

\section{PROCEDIMENTOS ÉTICOS E ADMINISTRAÇÃO DA PESQUISA:}

Todos os algoritmos abraçados, nesta pesquisa, seguiram as direções postergadas na Resolução 466/2012 do CNS e na Resolução 016/2000 do Conselho Federal de Psicologia para as pesquisas com indivíduos humanos (Conselho Nacional de Saúde [CNS], 2012; Associação Nacional de Pesquisa e Pós-Graduação em Psicologia [ANPEPP], 2000).

O aparelho da observação foi aplicado aos trabalhadores em todas as capitais brasileiras, de forma individual, por meio de um formulário eletrônico hospedado no google.docs.

De acordo com a disponibilidade do tempo e espaço físico dos respondentes em seus setores de trabalho ou fora dele, os mesmos, eram convidados a participar de forma voluntária, anônima e privada em responder o questionário eletrônico, o qual foi encaminhado para o e-mail, redes sociais etc. Foi informado aos que desejavam participar, quando acessaram o endereço da pesquisa, que a pesquisa será anônima e sigilosa, podendo desistir a qualquer momento dela, sem prejuízo algum para ele ou para o pesquisador. 


\section{RECIMA21 - REVISTA CIENTÍFICA MULTIDISCIPLINAR} ISSN 2675-6218

\section{ANÁLISE DE DADOS}

Em relação à análise dos dados, utilizar-se-á o pacote estatístico SPSSWIN, em sua versão 24.0, para tabular os dados e realizar as análises estatísticas descritivas (média e desvio padrão) e os cálculos referentes ao alfa de Cronbach, correlação de Pearson, teste t de Student e Anova.

No que se refere a primeira análise (Média e desvio padrão) são exclusivamente, medidas de dispersão com objetivo de avaliar a regularidade de um conjunto de dados relativo à amostra coletada; com elas é possível identificar o quanto os resultados não apenas poderiam assumir uma distribuição norma, bem como, a variação dos respondentes nas escalas de respostas (Dancey \& Reidy, 2006; Bisquerra; Sarriera \& Martinez, 2004).

De forma geral, ao utilizar o alfa neste estudo, está avaliando a fidedignidade da escala, isto é, busca-se a característica psicométrica que a medida utilizada deve possuir na qual, segundo o autor supracitado, ao mensurar o fenômeno estudado com os mesmos sujeitos ou outros em ocasiões diferentes, para que seja garantido a sua precisão instrumental, exige-se que apresente um coeficiente próximo a 1.

Com isso, o "Alfa de Cronbach (a) é um dos apontadores psicométricos mais utilizados para verificar a fidedignidade ou validade interna do instrumento, o qual deverá apresentar um alfa igual a 1 ; quanto mais próximo estiver desse número, melhor será sua precisão. Isso significa que os itens são homogêneos em sua mensuração e produzem a mesma variância, caracterizando uma segurança para a medida do fenômeno que se quer avaliar (Formiga, 2003, p. 134-135).

Em relação à correlação de Pearson, este cálculo é representado por um coeficiente ( $r$ ) indicando a relação produto-momento de mais de duas variáveis quantitativas, medindo o grau da correlação linear entre elas.

Trata-se de um índice adimensional, com valores situados ente -1,0 e 1,0 inclusive, que reflete a intensidade de uma relação linear entre dois conjuntos de dados; assim, $r=1$ Significa uma correlação perfeita positiva entre as duas variáveis, $r=-1$ Significa uma correlação negativa perfeita entre as duas variáveis - Isto é, se uma aumenta, a outra sempre diminui e $r=0$, significa que as duas variáveis não dependem linearmente uma da outra (Bisquerra; Sarriera \& Martinez, 2004; Figueiredo Filho \& Silva Junior, 2009).

Quanto ao teste t de Student, de acordo com Bisquerra, Sarriera \& Martinez (2004), tem diversas variações de aplicação, especialmente, para comparar duas (e somente duas) verificação dos escores médios em grupos diferentes, com o alvo de testar hipóteses entre eles. Por fim, em afinidade a apreciação de variância, ela é conhecida por permitir avaliar a comparação de médias em vários níveis de um ou mais tratamentos usando distribuições baseadas na teoria normal nas análises de construto. 


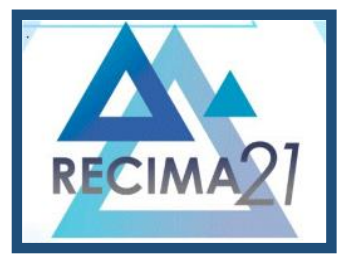

\section{RECIMA21 - REVISTA CIENTÍFICA MULTIDISCIPLINAR ISSN 2675-6218}

Ela é utilizada para constatar, doravante da formulação de um teste estatístico, a comparação de vetores de médias, com base na partição do total da variância em: variância devido ao efeito de tratamentos e variância devido ao erro. Em pesquisa que envolvem variáveis aleatórias contínuas, medidas na mesma unidade de pesquisa, pode-se pressupor a multinormalidade e realizar uma análise multivariada (Dancey \& Reidy, 2006; Hair et. al., 2009).

De acordo com os autores supracitados, um ponto relevante da análise multivariada é o aproveitamento da informação conjunta das variáveis envolvidas. As pressuposições para realização da MANOVA são as seguintes: 1) Modelo aditivo para efeitos de tratamentos, blocos (se houver) e erro; 2) Independência dos erros; 3) Igualdade da matriz de covariância $\Sigma$ para todas as amostras; 4) Distribuição multinormal dos erros, com variância do somatório dos construtos.

\section{RESULTADOS}

No que se refere às informações sobre a amostra, esta apresentou as seguintes características: 208 trabalhadores distribuídos em organizações públicas e privadas na cidade de Natal-RN, homens (41\%) e mulheres (59\%), com idades de 24 a 77 anos ( $M=43,69$, d.p. $=$ $11,18), 64 \%$ eram casados, tendo todos eles indicado ter acima de um salário-mínimo.

Antes de atender aos objetivos estabelecidos no artigo, foram realizadas análises estatísticas referentes à qualidade da amostra; desta maneira, em relação aos dados omissos das pesquisas, observou-se que eles estiveram abaixo do percentual de $5 \%$. Considerando esta informação, é possível substituí-los pela média ou moda dos dados da amostra, caso exista algum espaço vazio ou duplicidade de digitação na grade de inserção dos dados no programam estatístico (cf. Tabachnick \& Fidell, 2001).

Com relação à multicolinearidade entre as variáveis, as correlações entre elas estiveram dentro dos parâmetros definidos por Tabachnick \& Fidell (2001) [r $\leq 0,90$, variando de 0,32 a 0,69], revelando não existir variáveis com muita correlação, o que permite gerar modelos com baixo erro de medida. Verificou-se a presença de outliers multivariados na amostra; isto foi realizado através do teste de normalidade de Kolmogorov-Smirnov (KS), destinado a análise de amostras superiores a 100 sujeitos, observou-se uma normalidade $(K S=0,76)$ da amostra a um $p<0,58$.

Foi também avaliada a consistência interna dos construtos; para isso, realizou-se 0 cálculo do alfa de Cronbach ( $\alpha$ ) e de correlação intraclasse para ambas as escalas, tanto para pontuação total, quanto para as dimensões de cada uma delas, para as quais, seguiu-se a mesma pela distribuição fatorial proposta pelos autores das medidas (Pais, 2014; Siqueira, 2008) utilizadas no desenho.

Destaca-se que o cálculo referende-se do alfa é um dos indicadores psicométricos mais utilizados para avaliar a consistência ou validade interna de uma medida psicológica, social ou educacional. $O$ indicador alfa é aceito a partir de um valor mínimo $(0,70)$ a máximo $(1,00)$, quanto 


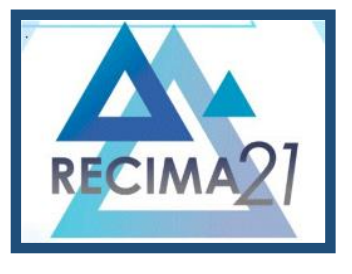

\section{RECIMA21 - REVISTA CIENTÍFICA MULTIDISCIPLINAR ISSN 2675-6218}

mais próximo de 1,00, melhor a precisão do que se pretende mensurar. Isso significa que os valores observados nestas análises indicam que os itens são homogêneos em sua mensuração, produzindo a mesma variância, podendo afirmar que a escala é segura para a medida do fenômeno que se quer avaliar (Pasquali, 2011; Kline, 2014).

Além de o indicador alfa oferecer maior garantia psicométrica para as referidas medidas, utilizou-se associado ao alfa, a correlação intraclasse (ICC); trata-se de um coeficiente de reprodutibilidade $(R)$, sendo com isso, uma estimativa da fração da variabilidade total de medidas devido a variações entre os indivíduos, isto é, o quanto esse indicador prevê, para pesquisas futuras, uma constante de uso da escala com amostras assumindo semelhantes categorias, a partir de procedimentos altamente standardizados e métodos de recolha de dados conhecidos (Dancey \& Reidy, 2006; Bisquerra, Sarriera \& Martinez, 2004; Matos, 2014).

Tabela 1: Escores dos alfas de Cronbach $(\alpha)$ das escalas.

\begin{tabular}{|c|c|c|c|c|}
\hline \multirow{2}{*}{$\begin{array}{l}\text { Construtos/ } \\
\text { Variáveis }\end{array}$} & \multicolumn{3}{|c|}{ Alfa de Cronbach } & \multirow{2}{*}{$\begin{array}{c}\text { ICC } \\
\text { (IC 95\%) }\end{array}$} \\
\hline & $\alpha$ geral & Voltem excluído & $\begin{array}{c}\mathrm{F} \\
\text { Friedman }\end{array}$ & \\
\hline $\begin{array}{l}\text { Percepção do Suporte } \\
\text { Organizacional }\end{array}$ & $0,87^{*}$ & $0,81-0,88$ & $28,89^{\star}$ & $\begin{array}{c}0,87^{*} \\
(0,81-0,90)\end{array}$ \\
\hline Gestão do conhecimento & $0,89^{*}$ & $0,87-0,92$ & $40,15^{\star}$ & $\begin{array}{c}0,89^{*} \\
(0,85-0,91)\end{array}$ \\
\hline Prática informal & $0,85^{*}$ & $0,78-0,86$ & $34,60^{*}$ & $\begin{array}{c}0,85^{*} \\
(0,80-0,85) \\
\end{array}$ \\
\hline Orientação Cultural & $0,87^{*}$ & $0,82-0,89$ & $22,75^{\star}$ & $\begin{array}{c}0,85^{*} \\
(0,80-0,87)\end{array}$ \\
\hline $\begin{array}{l}\text { Orientação } \\
\text { Competitiva }\end{array}$ & $0,80^{*}$ & $0,78-0,84$ & $29,35^{\star}$ & $\begin{array}{c}0,80^{*} \\
(0,78-0,82)\end{array}$ \\
\hline Prática Formal & $0,84^{*}$ & $0,725-0,85$ & $35,25^{\star}$ & $\begin{array}{c}0,81^{*} \\
(0,80-0,83)\end{array}$ \\
\hline
\end{tabular}

Notas: Elaborado pela autora. $\mathrm{V} \alpha=$ Variação alfa quando 0 item for excluído; $\mathrm{F}=$ Teste de Friedman; ICC = Correlação intraclasse. ${ }^{*} p<0,001$.

Assim considerado, na tabela 1, com base nos indicadores apresentados, é possível destacar que as escalas utilizadas são confiáveis. Considerando que o constructo tanto da percepção do suporte organizacional quanto da gestão do conhecimento, foram consistentes em sua estrutura fatorial e em termos da definição destes referentes aos seus fatores, resultados, os quais, atendem aos princípios estatísticos de qualidade psicométrica destinados às escalas de medidas (Bisquerra, Sarriera \& Martinez, 2004; Kline, 2014; Matos, 2014; Fantinelli, 2018).

Destaca-se também o ICC, seja considerando a pontuação total, seja na distribuirção fatorial, este indicador estatístico revelou a possibilidade de que poderá encontrar resultados próximos aos achados no presente estudo, se e somente se, verificá-los numa amostra semelhante à coletada para esta disertação (Silva, Velo \& Pereira, 2016). Os resultados do ICC, na concepção dos autores supracitados sugere que a reprodutibilidade de um estudo 


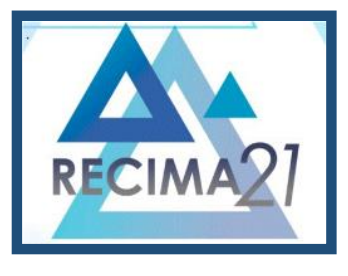

\section{RECIMA21 - REVISTA CIENTÍFICA MULTIDISCIPLINAR ISSN 2675-6218}

(especialmente, das escalas) na categoria empírica e teórica destacada na dissertação, tanto é viável, quanto possibilita condições preditivas de resultados próximos aos encontrados na dissrtação.

Sendo assim, provavelmente, esses instrumentos poderão ser utilizados em outras pesquisas de trabalhadores que contemplem as características sociodemográficas observadas no presente estudo. Um fato que chama atenção é o quanto serem esses indicadores psicométricos utéis, não apenas para que os construtos abordados sejam confirmados e tornem os conceitos, respectivamente, elaborados, por Pais (2014) relativo a gestão do conhecimetno e por Siqueira (2008) para a percepção do suporte organizacional e viáveis para uma avaliação psicológica nas organizações, bem como, conduzir pesquisas diagnósticas e interventivas na àrea do comportamento organizacional (Abbad, Loiola, Zerbine, \& Borges-Andrade, 2013; Azevedo \& Cruz, 2006). Com as escalas demonstrando consistência na avaliação dos construtos apresentados, avaliou-se o objetivo principal da dissertação, apresentandos a seguir.

A partir das análises estatísticas apresentadas acima, procurou-se atender ao objetivo principal desta dissertação (verificar a influência da impulsividade sobre a atitude de endividamento); sendo assim, realizou-se uma análise correlação de Pearson a fim de avaliar quais são dos construtos e suas respectivas dimensões relacionam-se com a percepção do suporte organizacional.

Na tabela 2, poderá observar, especificamente, que tanto houve uma relação positiva do suporte organizacional e a gestão do conhecimento, bem como, com as dimensões deste construto. Desta maneira, seja considerando a pontuação da gestão do conhecimento, seja nas dimensões que avaliam a orientação e da prática desta gestão, foram todas significativas. Algo que merece ser destacado é, também, no que diz respeito a cada item que forma o construto do suporte organizacional, os quais, também, estiveram correlacionados e significativos.

Tabela 2: Escores correlacionais entre suporte organizacional e gestão do conhecimento em

\begin{tabular}{|c|c|c|c|c|c|}
\hline \multirow[t]{2}{*}{$\mathrm{VI}$} & \multirow[b]{2}{*}{$\mathrm{GC}_{\text {total }}$} & \multicolumn{2}{|c|}{ Orientação da GC } & \multicolumn{2}{|c|}{ Prática da GC } \\
\hline & & $\begin{array}{c}\text { Orientação } \\
\text { cultural }\end{array}$ & $\begin{array}{l}\text { Orientação } \\
\text { competência }\end{array}$ & Prática Formal & Prática Informal \\
\hline SOrg total & $0,39^{*}$ & $0,31^{*}$ & $0,39^{*}$ & $0,40^{\star}$ & $0,31^{*}$ \\
\hline $\mathrm{SO} 1$ & $0,21^{*}$ & $0,38^{*}$ & $0,17^{*}$ & $0,37^{*}$ & $0,15^{\star}$ \\
\hline SO2 & $0,22^{*}$ & $0,29^{*}$ & $0,15^{\star}$ & $0,22^{*}$ & $0,15^{\star}$ \\
\hline $\mathrm{SO} 3$ & $0,24^{*}$ & $0,40^{*}$ & $0,20^{*}$ & $0,45^{\star}$ & $0,23^{*}$ \\
\hline $\mathrm{SO} 4$ & $0,29^{*}$ & $0,52^{*}$ & $0,33^{*}$ & $0,57^{*}$ & $0,25^{\star}$ \\
\hline SO5 & $0,25^{*}$ & $0,40^{*}$ & $0,31^{*}$ & $0,52^{*}$ & $0,26^{\star}$ \\
\hline SO6 & $0,32^{*}$ & $0,37^{*}$ & $0,26^{*}$ & $0,43^{*}$ & $0,17^{*}$ \\
\hline SO7 & $0,34^{*}$ & $0,47^{*}$ & $0,32^{*}$ & $0,56^{*}$ & $0,29^{*}$ \\
\hline SO8 & $0,25^{*}$ & $0,20^{*}$ & $0,16^{*}$ & $0,27^{*}$ & $0,13^{*}$ \\
\hline SO9 & $0,43^{*}$ & $0,45^{*}$ & $0,24^{*}$ & $0,24^{*}$ & $0,30^{*}$ \\
\hline
\end{tabular}




\section{RECIMA21 - REVISTA CIENTÍFICA MULTIDISCIPLINAR ISSN 2675-6218}

Nilton Soares Formiga, Juliana Bianca Maia Franco, Sarah Ruth Araújo Grangeiro, Heitor César Costa Oliveira, lonara Dantas Estevam

Notas: * $p<0,01$. SOrgtotal = pontuação total do Suporte Organizacional. GCtotal = Pontuação geral da Gestão do Conhecimento.SO1 = Esta empresa ignoraria qualquer reclamação de minha parte; SO2 = Esta empresa não considera meus interesse quando toma decisões que me afetam; SO3 = É possível obter ajuda desta empresa quando tenho um problema; $\mathrm{SO} 4$ = Esta empresa realmente preocupa-se com meu bem-estar; SO5= Esta empresa estaria disposta a ampliar suas instalações para me ajudar a utilizar minhas melhores habilidades no desempenho do meu trabalho; SO6 = Esta empresa está pronta a ajudar-me quando eu precisar de um favor especial; SO7 = Esta empresa preocupa-se com minha satisfação no trabalho; SO8 = Esta empresa preocupa-se mais com seus lucros do que comigo; SO9 = Esta empresa tentar fazer com que meu trabalho seja o mais interessante possível.

Com base nestas correlações, realizou-se uma ANOVA univariada, a fim de avaliar o quanto a gestão do conhecimento é influenciada pelo suporte organizacional; a título de parcimônia, optou-se pela organização do construto da gestão do conhecimento em termos de sua análise por meio das práticas (Formal e Informal) e das orientações (Cultural e Competência) da gestão do conhecimento.

$\mathrm{Na}$ tabela 3, pode-se observar que a gestão do conhecimento, seja na prática ou na orientação, está associado ao maior nível de suporte organizacional, tendo todos os escores médios encontrados neste nível, sendo significativos. Em termos de comparações múltiplas, a partir do teste de Sheffé, observou-se a seguinte hierarquia significativa: $c>b>a$, na alta percepção do suporte organizacional, corroborando o que se esperava, isto é, quanto maior o suporte organizacional, maior a gestão do conhecimento.

Tabela 3: Análise de variância na gestão do conhecimento (prática e orientação) em função do nível de percepção do suporte organizacional.

\begin{tabular}{|c|c|c|c|c|c|c|}
\hline \multirow[t]{2}{*}{$\mathrm{VI}$} & \multirow{2}{*}{$\begin{array}{c}\text { VD } \\
\text { (Nível de Suporte) }\end{array}$} & \multirow[t]{2}{*}{ Média } & \multirow[t]{2}{*}{ d.p. } & \multicolumn{3}{|c|}{ Estatística } \\
\hline & & & & $\mathrm{F}$ & gl & $p$-valor \\
\hline \multirow{4}{*}{$\begin{array}{l}\text { Gestão do } \\
\text { Conhecimento }\end{array}$} & Baixo $^{(a)}$ & 76,18 & 13,98 & \multirow{4}{*}{20,07} & \multirow{4}{*}{2} & \multirow{4}{*}{0,001} \\
\hline & Moderado(b) $^{(b)}$ & 81,47 & 12,92 & & & \\
\hline & Alto $^{(c)}$ & 89,97 & 12,43 & & & \\
\hline & Total & 82,70 & 14,31 & & & \\
\hline \multirow{4}{*}{$\begin{array}{l}\text { Prática da Gestão } \\
\text { do Conhecimento }\end{array}$} & Baixo(a) & 33,14 & 6,60 & \multirow{4}{*}{21,60} & \multirow{4}{*}{2} & \multirow{4}{*}{0,001} \\
\hline & Moderado $^{(b)}$ & 36,09 & 6,91 & & & \\
\hline & Alto & 40,46 & 6,61 & & & \\
\hline & Total & 36,64 & 7,35 & & & \\
\hline \multirow{4}{*}{$\begin{array}{l}\text { Orientação da } \\
\text { Gestão do } \\
\text { Conhecimento }\end{array}$} & Baixo $^{(a)}$ & 37,77 & 7,93 & \multirow{4}{*}{18,63} & \multirow{4}{*}{2} & \multirow{4}{*}{0,001} \\
\hline & Moderado $^{(b)}$ & 41,67 & 7,12 & & & \\
\hline & Alto $^{(c)}$ & 45,24 & 6,85 & & & \\
\hline & Total & 41,61 & 7,932 & & & \\
\hline
\end{tabular}

Por fim, realizou-se um teste $\mathrm{t}$ de Student, comparando as pontuações médias no suporte organizacional e na gestão do conhecimento em relação às organizações públicas e privadas; observou-se que os respondentes das organizações privadas apresentaram maiores escores na variável percepção do suporte organizacional $(\mathrm{t}=-2,16, \mathrm{gl}=196,28$, $\mathrm{p}$-valor $<0,05)$, 


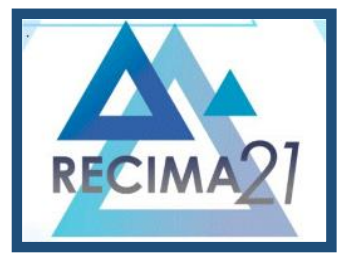

\section{RECIMA21 - REVISTA CIENTÍFICA MULTIDISCIPLINAR ISSN 2675-6218}

na gestão do conhecimento $(\mathrm{t}=-2,62, \mathrm{gl}=191,31, \mathrm{p}$-valor $<0,05)$ e na dimensão orientação a competitividade da $\mathrm{GC}(\mathrm{t}=-2,19, \mathrm{gl}=181,33, \mathrm{p}$-valor $<0,05)$.

\section{DISCUSSÃO}

De forma geral, o presente estudo se insere no campo dos estudos da psicologia organizacional e do trabalho e teve como objetivo principal verificar a influência do suporte organizacional sobre a gestão do conhecimento em trabalhadores de organizações públicas e privadas na cidade de Natal-RN.

Inicialmente, procurou-se atender ao objetivo específico relativo à qualidade das escalas de medidas; a razão a que se deveu essa atividade refere-se à realização de uma busca nos sites da produção científica sobre o tema, tendo encontrado de pouca a nenhuma produção com as escalas de medidas utilizadas na dissertação.

Sendo assim, a análise de consistência interna, com base nos alfas e no ICC, confirmou a qualidade das medidas de suporte organizacional e gestão do conhecimento, podendo, com isso, afirmar que as medidas-construtos destinadas a pesquisa quantitativa nesta dissertação é segura e atendeu aos princípios psicométricos corroborando a hipótese levantada (isto é, a de que tais escolas poderiam ser consistentes, de acordo com o que sugere os autores supracitados desta medidas [cf. Formiga \& Souza, 2019; Pais, 2014).

Em relação a proposta correlacional elaborada, a qual esperava-se existir relação positiva e significativa entre o suporte organizacional e gestão do conhecimento. Desta maneira, observando a tabela 2, tanto as correlações do construto suporte organização quanto os itens que o compõem se relacionaram a gestão do conhecimento e suas respectivas dimensões da orientação e da prática da gestão, as quais, todas sendo significativas; condição que corrobora os pressupostos teóricos deste trabalho.

Com isso, ao considerar os resultados destacados refletidos no parágrafo acima, isto permite inferir, de forma parcimoniosa, que provavelmente, a gestão do conhecimento tem seu melhor desenvolvimento, se e somente se, estiver pautada numa dinâmica organizacional que influencie na maneira como o trabalhador percebe que a sua organização apoia, gera vínculo, valora a sua função e o mesmo quanto trabalhador, podendo contribuir para o desenvolvimento de uma autoestima e até em práticas de inovação (cf. Siqueira, 2008; Formiga \& Freire, 2018; Formiga, Freire, Batista \& Estevam, 2018; Formiga, Araújo, Pastana, Miranda, \& Mafra, 2019; Formiga, Freire \& Fernandes, 2020).

Apesar de não existir estudos que contemplem, neste conjunto de correlações abordados nesta dissertação, é possível observar o quanto os resultados apresentados convergem para os estudos que abordam de forma específica a importância do suporte organização nas experiências dos trabalhadores, por exemplo: no estudo desenvolvido por Formiga e Freire (2019), avaliando o quanto suporte organização interfere na expectativa de vida dos trabalhadores, foi observado que tal construto contribui muito o desenvolvimento de estratégias de gestão pessoal, a qual, tinha seu 


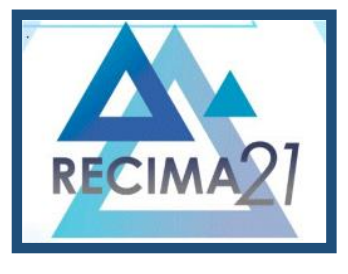

\section{RECIMA21 - REVISTA CIENTÍFICA MULTIDISCIPLINAR ISSN 2675-6218}

foco na avaliação preditiva na relação trabalho-emprego-produtividade, gerindo assim, no trabalhador a busca de oportunidades reais entre o que se faz e o que possível fazer nas organizações, isto é, quanto maior suporte organizacional, melhor as expectativas por melhores condições da sociedade, do sucesso profissional e financeiro, afinal, trabalho é, atualmente considerado também, uma forma de consumo.

No estudo desenvolvido por Almeida, Formiga, Junior, Sousa, (2020), sobre gestão do conhecimento e oportunidade de aprendizagem organizacional na condução dos resíduos dos serviços de saúde nos hospitais de referências em quimioterapia, revelou não apenas que o gerir conhecimento é capaz de influenciar na forma de aprendizagem organizacional por parte do funcionário, denotando que não se trata simplesmente de uma pensar e/ou saber a respeito de algo, mas, que esta condição permitirá ao trabalhador se orientar cognitivamente, em termos de suas práticas profissionais e de segurança em saúde.

Tal fato, também foi comprovado que se avaliou a capacitação dos funcionários, a qual, por si só, não era suficiente, e que esta deveria estar associada à gestão e ao processo de aprendizagem organizacional, assim, observou-se que aquele profissional que teve menor capacitação, apresentou menor escore no construto da gestão do conhecimento. E mais! Ainda no estudo deles, quando se avaliou os encontros periódicos com a chefia, maior foi a frequência dos escores na gestão do conhecimento

Assim, reflete-se que a gestão, não apenas é útil, mas que, tem sua expertise e qualidade quanto associada ao suporte organizacional. Não basta, por si, existir um processo de gestão, mas, acredita-se que este, deverá se associar aos processos de incorporação de inovações práticas e de orientação da gestão do conhecimento, os quais, são necessários frente a competitividade e a dinamicidade funcional no processo organizacional. Tais reflexões, suscitam atualização e acompanhamento de tendências do mercado, incorporação de medidas que valorizem e tenham responsabilidade com a qualidade do serviço (Formiga, Silva, Silva, Firmino, Santos, Azevedo \&Martins, 2020).

De forma geral, ao considerar essas relações, salienta-se que a gestão do conhecimento não venha ocorrer de maneira independente e no vazio organizacional em termos de simples variável para formação, tomada de decisão e soluções de problemas de gestão humana, mas, seria muito importante avaliar o quanto a própria organização apoia e valoriza o seu funcionário, isto é, oferece suporte organizacional, condição a qual, acredita-se, que poderá ser estabelecida pelo Recursos Humanos das organizações.

A reflexão acima é bem pontual, principalmente quando se remete à avalição da Análise de Variância entre os construtos destacada na tabela 3. Assim como se sugeria, quanto maior foi o escore no suporte organizacional, maior o escore na gestão do conhecimento; esta proposta foi corroborada, pois permite afiram de forma segura os resultados correlacionais, pois, o maior 


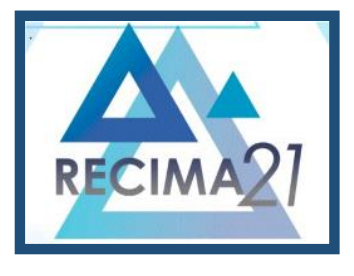

\section{RECIMA21 - REVISTA CIENTÍFICA MULTIDISCIPLINAR ISSN 2675-6218}

escore no suporte organizacional corresponde à gestão do conhecimento, bem como as suas dimensões da prática e da orientação.

Por fim, um resultado que chamou atenção foi quanto a comparação entre os respondentes das organizações públicas e privadas estarem apenas para o suporte organização, a gestão do conhecimento quanto pontuação total e a orientação a competitividade, nas quais a organização privada apresentou maiores escores.

Com isso, os resultados apresentados nesta dissertação têm a sua importância não apenas para refletir práticas para o recurso humano $(\mathrm{RH})$, mas também de política organizacional de produtividade e qualidade de vida do trabalhador em seu ambiente labora. Ao enfatizar em estudos e práticas sobre a influência destas variáveis (gestão e suporte organizacional) sugere que, quando se pretender avaliar o desenvolvimento e manutenção da qualidade, inovação e competitividade na dinâmica organização-trabalhador, que seja refletido e aplicada condições práticas e de orientação quanto à importância da organização como um todo e de seus gestores frente à valoração do próprio funcionário.

Com isso, salientar um proposta com base nas concepções das dimensões sociocognitivas dos aspectos micro-organizacionais, especialmente, quanto a construção e manutenção da percepção que o trabalhador tem sobre a organização, pois esta é construída sob via de mão dupla, trata-se de identificar os possíveis entraves relativo ao 'não-saber' e/ou 'não-aprender' de atividades laborais importantes e das lacunas organizacionais frente às atitudes de inovação e competividade produtivas do trabalhador.

Desta maneira, na proposta central destes resultados, constatou-se que a organização e sua dinâmica funcional tem um papel importante na ação dos seus gestores (gerar, gerir e manter uma produtividade) na qualidade inovadora e de satisfação com o trabalho por parte dos funcionários e, também, no caso de inexistência ou insuficiência de uma política organizacional sobre este tema em questão, é crucial que o próprio trabalhador busque meios para que se mantenha informado, para concorrência e manutenção da sua atividade laboral

\section{CONSIDERAÇÕES FINAIS}

A presente pesquisa buscou analisar as representações sociais sobre trabalho para pequenos e grandes produtores rurais do município de Diamantino - MT. Foi possível perceber, por meio dos relatos apresentados pelas entrevistadas, elementos significativos que possibilitaram a aproximação com a realidade de vida de cada um dos participantes, onde houveram relatos sobre as dificuldades e superações relacionadas ao trabalho e vinculações com a permanência no trabalho rural.

O trabalho está associado à Dignidade e Realização humana, sendo benéfico para a saúde e qualidade de vida, organiza a rotina e ocupa lugar central na vida dos indivíduos, 


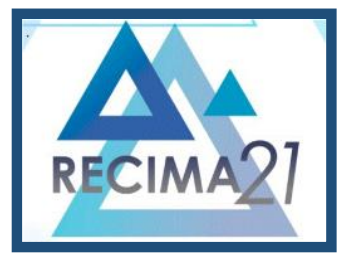

\section{RECIMA21 - REVISTA CIENTÍFICA MULTIDISCIPLINAR ISSN 2675-6218}

possibilita a sobrevivência, acúmulo de bens, dignidade e realização, sendo que os indivíduos se autodeterminam para sua realização.

Assim, pode-se dizer que o trabalho, segundo os entrevistados, tem caráter positivo relacionado à Dignidade e Realização. Por meio do trabalho os indivíduos idosos podem garantir sua subsistência, adquirindo alimentos e pagando as contas. Os idosos reconhecem que há limitações na sua capacidade para executar as tarefas, no entanto, associam o movimento físico na execução das tarefas à boa saúde, e ainda assim se sentem otimistas e úteis por continuar fazendo algumas coisas (cuidar da horta e dos animais), afastando atividades perigosas ou que exigem muito esforço físico.

Com relação à rotina, o trabalho é visto como central na vida dos indivíduos, pois como sempre trabalharam, este organiza a rotina e dá sentido à sua existência e utilidade enquanto pessoa. O trabalho ainda é percebido como forma de estabelecer contato social e obter merecimento, pelo esforço e dedicação, melhorando a autoestima e engrandecendo o ser humano. Desta forma, a continuidade no trabalho, afasta os aspectos negativos do envelhecimento e dá aos idosos a impressão de não terem envelhecido.

Para que possam continuar trabalhando, alguns idosos relatam Autodeterminação, pois com as limitações impostas pelo envelhecimento, a decisão de continuar trabalhando, e não ficar esperando passivamente que "caia do céu", é expressa como coragem por parte do idoso. Os idosos são otimistas sobre o trabalho, qualificando como lazer e diversão, no entanto, por vezes trabalham e cuidam da propriedade sozinhos, seja pela viuvez, seja porque os filhos adultos trabalham em outros lugares.

O trabalho também esteve associado às necessidades humanas básicas, seja para sobrevivência, seja como contribuição para a saúde. Justificam que sem o trabalho, não alcançam saúde, e não fazem aquisições monetárias. Por fim, o otimismo está relacionado com todos os aspectos positivos do trabalho, como a moralidade, honestidade e saúde.

\section{REFERÊNCIAS}

ABBAD, G. S.; LOIOLA, E.; ZERBINE, T.; BORGES-ANDRADE, J. Aprendizagem em Organizações e no Trabalho. In.: Borges, L. O.; Mourão, L. (Orgs.). O Trabalho e as Organizações - Atuações a partir da Psicologia. Porto Alegre: Artmed, 2013. Vol. 1, p. 497-527.

ALMEIDA, L. A. L.; FORMIGA, N. S.; A. JUNIOR, J. I.; SOUSA, P. M. Quanto mais se sabe, mais segurança tem! A importância da gestão do conhecimento na condução dos resíduos sólidos de quimioterapia. Revista Psicologia e Saúde em Debate, v. 6, n. 1, p. 430-442, 2020.

AZEVEDO, Beatriz Marcondes de; CRUZ, Roberto Moraes. O processo de diagnóstico e de intervenção do psicólogo do trabalho. Cadernos de Psicologia Social do Trabalho, v. 9, n. 2, p. 89-98, 2006.

AZEVEDO, I. Análise e aplicação bibliométrica na administração e áreas afins: um levantamento nacional. Revista InterScientia, v. 6, n. 2, p. 146-164, 2018. 


\section{RECIMA21 - REVISTA CIENTÍFICA MULTIDISCIPLINAR} ISSN 2675-6218

BERTONCELLO, B.; BORGES-ANDRADE, J. E. Relações entre saúde mental do trabalhador e suporte organizacional. Revista Laborativa, v. 4, n. 2, p. 85-102, 2015.

BISQUERRA, R.; SARRIERA, J. C.; MARTÍNEZ, F. Introdução à estatística: enfoque informático com o pacote estatístico SPSS. Porto Alegre: Artmed, 2004.

BORGES-ANDRADE, G.; ABBAD, L. Mourão. Treinamento, desenvolvimento e educação em organizações e trabalho: fundamentos para a gestão de pessoas. Porto Alegre: Artmed, 2006.

BRASIL. Ministério da Saúde. Conselho Nacional de Saúde. Resolução n. 196, de 10 de outubro de 1996. Aprova as diretrizes e normas regulamentadoras de pesquisas envolvendo seres humanos. Brasília: Diário Oficial da União, 16 out. 1996.

BRASIL. Ministério da Saúde. Conselho Nacional de Saúde. Resolução n. 466, de 12 de dezembro de 2012. Aprova diretrizes e normas regulamentadoras de pesquisas envolvendo seres humanos. Brasília: Diário Oficial da União, 12 dez. 2012.

COSTA, S. A. (2019). Planejamento financeiro pessoal: uma proposta para a saúde financeira do brasileiro da classe C. Porto: Universidade Fernando Pessoa Porto, 2019.

DANCEY, C. P.; REIDY, J. Estatística sem matemática para psicologia. Porto Alegre: Artmed, 2006.

EISENBERGER, R.; HUNTINGTON, R.; HUTCHINSON, S.; SOWA, D. Perceived organizational support. Journal of Applied Psychology, v. 71, p. 500-507, 1986.

FANTINELLI, A. A. Desenvolvimento e validação do instrumento específico para complexidade assistencial de puérperas e recém-nascidos: escala de Fantinelli. 2018. Tese (Doutorado) - Pontifícia Universidade Católica do Rio Grande do Sul, 2018.

FAUL, F.; ERDFELDER, E.; BUCHNER, A.; LANG, A.-G. Statistical power analyses using G*Power 3.1: tests for correlation and regression analyses. Behavior Research Methods, v. 41, p. 1149-1160, 2009.

FIGUEIREDO FILHO, D. B.; SILVA JUNIOR, J. A. Desvendando os Mistérios do Coeficiente de Correlação de Pearson (r). Revista Política Hoje, v. 18, n. 1, p. 115-146, 2009.

FORMIGA, N. S. Fidedignidade da escala de condutas anti-sociais e delitivas ao contexto brasileiro. Revista Psicologia em Estudo, v. 8, n. 2, p. 133-138, 2003.

FORMIGA, N. S.; ARAUJO, I. T.; PASTANA, S. T. G.; MIRANDA, A. L. B. B.; MAFRA, A. L. Escala de engajamento no trabalho: Evidência empírica da estrutura fatorial em trabalhadores brasileiros. Persperctivas em Psicologia, v. 23, n. 1, p. 119-122, 2019.

FANDIÑO, A. M.; FORMIGA, N. S.; MENEZES, R. M. Organizational social capital, resilience and innovation validation of a theoretical model for specialized workers. Journal of Strategy and Management, 2018.

FORMIGA, N. S.; FERNANDES, A. J.; FREIRE, B. G. O. et al. Organizational support, social capital and innovative attitudes: A correlational study among workers in the city of Natal-RN. Research, Society and Development, v. 10, n. 3, 2021.

FORMIGA, N. S.; FLEURY, L. F. O.; SOUZA, M. A. Evidências de validade da escala de percepção de suporte organizacional em funcionários de empresa pública e privada. Estudos Interdisciplinares em Psicologia, v. 5, n. 2, p. 60-76, 2014.

FORMIGA, N. S.; FREIRE, B. G. O. Correlatos entre suporte organizacional e expectativa de futuro em funcionários de organizações públicas e privadas no Brasil. Psicologia.com.pt, v. 1, n. 1, p. 1-18, 2018.

FORMIGA, N. S.; FREIRE, B. G. O.; AZEVEDO, I. M.; FARIA, S. S. Correlatos entre suporte organizacional, psicológico, capital no trabalho e expectativa de futuro: um estudo com trabalhadores brasileiros. Research, Society and Development, v. 9, n. 6, e155963486, 2020. 


\section{RECIMA21 - REVISTA CIENTÍFICA MULTIDISCIPLINAR ISSN 2675-6218}

FORMIGA, N. S.; FREIRE, B. G. O.; AZEVEDO, I. M. et al. Organizational support, psychological, capital at work and future expectancy in the Brazilian workers. Research, Society and Development, v. 10, n. 3, 2021.

FORMIGA, N. S.; FREIRE, B. G. O.; BATISTA, P. F. A.; ESTEVAM, I. D. Suporte organizacional e autoestima em funcionários de organizações públicas e privadas no brasil. Psicologia.com.pt, v. 1, n. 1, p. 1-15, 2017.

FORMIGA, N. S.; FREIRE, B. G. O.; FERNANDES, A. A medida da atitude de inovação: valores métricos, invariância fatorial e diferenças em trabalhadores. Revista de Carreiras e Pessoas, v. 10, n. 1, p. 177-198, 2020.

FORMIGA, N. S.; MIRANDA, A. L. B. B.; ARAUJO, I. T.; PASTANA, S. T. G.; MAFRA, A. L. Evidência da invariância fatorial e validade convergente da escala de suporte organizacional: estudo com trabalhadores brasileiros. Boletim da Academia Paulista de Psicologia, v. 38, n. 94 , p. 27-35, 2018.

FORMIGA, N. S.; SILVA, A. G. F.; SILVA, J. D. Transtorno emocional leve em trabalhadores: Verificação de um modelo teórico a partir do suporte organizacional, gestão do conhecimento e capital psicológico positivo. Research, Society And Development, v. 9, n. 1, p. 1-28, 2020.

FORMIGA, N. S.; SOUZA, I. M. A saúde laboral em trabalhadores administradores: uma explicação interacional entre variáveis macro, meso e micro-organizacional no Rio Grande do Norte. Beau Bassin: Novas Edições Acadêmicas, 2019. v. 1. 105 p.

GRANGEIRO, S. R. A.; FORMIGA, N. S. O trabalhador e os valores (in)tangíveis: um estudo sobre a percepção do suporte organizacional e gestão do conhecimento em trabalhadores de organizações públicas. Beau Bassin - Mauritius: Novas Edições Acadêmicas. 2021. 60 p.

HAIR, J.; ANDERSON, R.; TATHAM, R. E.; BLACK, W. Análise de Dados Multivariados. Boston: Editora McGraw Hill, 2009.

KLINE, C. E. The bidirectional relationship between exercise and sleep: Implications for exercise adherence and sleep improvement. Am J Lifestyle Med., v. 8, n. 6, p. 375-379, nov./dec. 2014.

LEITE, Andrea Ferreira; NOGUEIRA, Júlia Aparecida Devidé. Fatores condicionantes de saúde relacionados ao trabalho de professores universitários da área da saúde: uma revisão integrativa. Revista Brasileira de Saúde Ocupacional, v. 42, n. 6, 2017.

MATOS, Gustavo Gomes de. Comunicação empresarial sem complicação. 3. ed. Barueri: Manole, 2014.

MCINERNEY, D. M.; ALI, J. Avaliação multidimensional e hierárquica da motivação escolar: validação transcultural. Psicologia Educacional, v. 26, n. 6, p. 717-734, 2006. DOI: https://doi.org/10.1080/01443410500342559

MOHAPATRA, S.; AGRAWAL, A.; SATPATHY, A. Designing Knowledge Management Strategy. In.: Projetando Estratégias de Negócios Habilitadas para a Gestão do Conhecimento: gestão para profissionais. Cham: Springer, 2016.

MILES, J.; SHEVLIN, M. Applying Regression e Correlation: a guide for students and researchers. London: SAGE Publications, 2001.

MIRANDA, S. Como as necessidades de informação podem se relacionar com as competências informacionais. Ciência da Informação, v. 35, n. 3, p. 99-114, 2006.

PAIS, L. Gestão do conhecimento. In.: SIQUEIRA, M. M. M. (Org.). Novas medidas do comportamento organizacional: ferramentas de diagnóstico e de gestão. Porto Alegre: Artmed, 2014.

PASQUALI, L. Psicometria: teoria dos testes na Psicologia e na educação. 4. ed. Petrópolis/RJ: Vozes, 2011. 


\section{RECIMA21 - REVISTA CIENTÍFICA MULTIDISCIPLINAR ISSN 2675-6218}

CORRELATOS E VARIAÇÕES NA PERCEPCÃO DO SUPORTE ORGANIZACIONAL E GESTÃO DO CONHECIMENTO EM TRABALHADORES DE ORGANIZAČ̃ES PÚBLICAS E PRIVADAS EM NATAL-RN Nilton Soares Formiga, Juliana Bianca Maia Franco, Sarah Ruth Araújo Grangeiro, Heitor César Costa Oliveira, lonara Dantas Estevam

PEREIRA, Gabriela Aguiar; FORMIGA, Nilton S.; ESTEVAM, Ionara D. Análise confirmatória da medida do suporte organizacional em enfermeiros de um hospital público de uma capital do nordeste brasileiro. Boletim - Academia Paulista de Psicologia, v. 39, n. 96, p. 59-68, 2019.

PODSAKOFF, P. M.; SCOTT, B. M.; PODSAKOFF, N. P. Common method biases in behavioral research: A critical review of the literature and recommended remedies. Journal of Applied Psychology, v. 88, n. 5, p. 879-903, 2003.

SILVA, A. F.; VELO, M. M. A. C.; PEREIRA, A. C. Importância da reprodutibilidade dos métodos para diagnóstico em odontologia. RFO UPF, v. 21, n. 1, 2016.

SIQUEIRA, M. M. M. Novas medidas do comportamento organizacional: ferramentas de diagnóstico e de gestão. Porto Alegre: Artmed, 2014.

SIQUEIRA, M. M. M. Antecedentes de comportamentos de cidadania organizacional: análise de um modelo pós-cognitivo. 1995. 265f. Tese (Doutorado em Psicologia) - Instituto de Psicologia, Universidade de Brasília, Brasília, 1995.

SIQUEIRA, M. M. M.; PADOVAM, A. R. Bases teóricas de bem-estar subjetivo, bem-estar psicológico e bem-estar no trabalho. Psicologia: Teoria e Pesquisa, v. 24, p. 201-209, 2008.

TABACHNICK, B. G.; FIDELL, L. S. Using Multivariate Statistics. 4. ed. Boston: Allyn and Bacon, 2001.

TORRES, A. R. A.; ChAGAS, M. I. O.; MOREIRA, A. C. A.; BARRETO, I. C. D. H. C.; RODRIGUES, E. M. O adoecimento no trabalho: repercussões na vida do trabalhador e de sua família. SANARE-Revista de Políticas Públicas, v. 10, n. 1, p. 42-48, 2013.

ZANELLI, J.; BORGES-ANDRADE, E.; BASTOS, A. V. B. (Eds.). Psicologia, organizações e trabalho no Brasil. Porto Alegre: Artmed, 2004. p. 466-491. 